\title{
Calorimetric study of the nematic to smectic-A phase transition in octylcyanobiphenyl-hexane binary mixtures
}

\author{
Krishna P. Sigdel and Germano S. Iannacchione* \\ Department of Physics, Worcester Polytechnic Institute, \\ Worcester, Massachusetts 01609, USA
}

(Dated: November 11, 2018)

\begin{abstract}
The continuous nematic to smectic- $A(N-\operatorname{Sm} A)$ phase transition has been studied by highresolution ac-calorimetry in binary mixtures of the liquid crystal octylcyanobiphenyl(8CB) and a non-mesogenic, low-molecular weight, solvent n-hexane(hex) as a function of temperature and solvent concentration. Heating and cooling scans about the $N$-Sm $A$ transition temperature were repeatedly performed on pure and six $8 \mathrm{CB}$ +hex samples having hexane molar concentration ranging from $x_{h e x}=0.02$ to 0.12 . All $8 \mathrm{CB}+$ hex samples in this range of $x_{\text {hex }}$ remain macroscopically miscible and exhibit an $N$-Sm $A$ heat capacity peak that shifts non-monotonically to lower temperature and evolves in shape, with a reproducible hysteresis, as $x_{h e x}$ increases. The imaginary part of heat capacity remains zero up to $x_{h e x}^{T C P} \simeq 0.07$ above which the distinct peak is observed, corresponding to a jump in both the real and imaginary enthalpy. A simple power-law analysis reveals an effective exponent that increases smoothly from 0.30 to 0.50 with an amplitude ratio $A^{-} / A^{+} \rightarrow 1$ as $x_{\text {hex }} \rightarrow x_{\text {hex }}^{T C P}$. This observed crossover towards the $N$-Sm $A$ tricritical point driven by solvent concentration is consistent with previous results and can be understood as weakening of the liquid crystal intermolecular potential promoting increased nematic fluctuations.
\end{abstract}

PACS numbers: 61.30.-v, 64.70.mj, 65.40.Ba

* electronic address: gsiannac@wpi.edu 


\section{INTRODUCTION}

Liquid crystals(LCs) are anisotropic fluids which exhibit a varieties of phases and phase transitions [1, 2]. The nematic and smectic- $A$ phases are the best known phases of liquid crystals. The transition between nematic $(N)$ and smectic- $A(\operatorname{Sm} A)$ phases is interesting and important because it involves the breaking of a continuous symmetry as well as sharing some properties with the superconducting transition in metals and the superfluid transition in ${ }^{4} \mathrm{He}$. The $N$-Sm $A$ transition is also a model phase transition for the study of confinement and disorder effects such as in mixtures with silica aerosil [3 5], or embedded in an aerogel [6, 7] and controlled porous glass [8 10]. The phase transition behavior is also sensitive to an applied external electric and magnetic field [11, 12] as well as with LC+LC mixtures [13-17]. Even though the $N$-Sm $A$ transition has been extensively studied [18], there remains many unresolved issues regarding the fundamental nature of the transition.

Recently, attention has been drawn to the study of miscible mixtures of liquid crystals and non-mesogenic, low-molecular weight, solvents for broadening the basic understanding of mesogenic order, critical behavior and tuning viscoelastic properties [19 23]. X-ray diffraction experiments performed on smectic- $A$ and smectic- $C$ thermotropic liquid crystals have demonstrated that the smectic layer spacing increases with the addition of organic solvents to the host liquid crystal indicating the formation of an organic lyotropic lamellar liquid crystal phase[23]. It was suggested from the visual inspection that for octylcyanobiphenyl (8CB) and n-hexane(hex) mixture systems ( having a volume fraction of $\geq 0.1$ ), the solvent is not uniformly distributed throughout the host LC and minimal, non-reproducible, swelling occurs. It was also suggested that the amount of solvent incorporated in a smectic liquid crystal depends on the host liquid crystal, nature and amount of solvent, and temperature; noting that the mixture phase separates for a solvent to liquid crystal mole ratio $\geq 1.0$. Other studies of the effect of a biphenyl solvent on the splay and bend elastic constant and the rotational viscosity coefficient of $8 \mathrm{CB}$ observed an anomalous behavior of $K_{11}$ and $\Delta \varepsilon$ near $N$-Sm $A$ transition[24]. A theoretical study on the influence of non-mesogenic solvent on the $N$-Sm $A$ phase transition using Landau approach found a concentration induced tricritical point for the $N$-Sm $A$ transition[21]. This theoretical model also found that the Frank elastic constants $K_{11}, K_{22}$, and $K_{33}$ are modified as a function of solvent concentration near the $N-\operatorname{Sm} A$ phase transition. 
A recent calorimetric study of the $N$-Sm $A$ transition in mixtures of $8 \mathrm{CB}$ and cyclo-hexane (8CB + chex) was performed under continuous stirring conditions [19]. This study found a linear decrease of the transition temperatures $T_{N A}$ with a linear increase of critical heat capacity exponent $\alpha$ with increasing mole fraction of cyclohexane $x_{\text {chex }}$. This behavior ends at a tricritical point (TCP) where the transition becomes first-order at $x_{\text {chex }}^{T C P}=0.046$, just below which $\alpha=0.5$ and the nematic range $\Delta T_{N}=T_{I N}-T_{N A}=4.8 K$. For $x_{\text {chex }}>x_{\text {chex }}^{T C P}$, the $N$-Sm $A$ latent heat smoothly increases non-linearly from zero [19].

The $N$-Sm $A$ phase transition is a non-trivial member of the 3D-XY universality class due to the anisotropy of its critical fluctuations parallel and perpendicular to the director [18, 25], 26]. The $N$-Sm $A$ critical behavior is strongly effected by the coupling between the smectic order parameter $\psi(\vec{r})=\psi_{0} \exp \left(i \overrightarrow{q_{0}} \cdot \vec{r}\right)$ and the nematic order parameter $Q_{i j}=$ $(1 / 2) S\left(3 \hat{n}_{i} \hat{n}_{j}-\delta_{i j}\right)$. Here, the $\psi$ is the amplitude of the one-dimensional density wave, $\rho(\vec{r})=\operatorname{Re}\left[\rho_{0}+\exp \left(i \overrightarrow{q_{0}} \cdot \vec{r}\right) \psi(\vec{r})\right], q_{0}=2 \pi / d$ is the wave vector corresponding to the layer spacing $d, S$ is a scalar parameter measuring the magnitude of orientational order on short length scales, and $\hat{n}$ is the nematic director describing spatial orientation of the orientational axis on longer length scales. It has been shown by de Gennes [1] and McMillan [27] that a mean-field coupling between $S$ fluctuations and smectic order $\delta S-\psi$ can drive a secondorder $N$-Sm $A$ phase transition first-order via a tricritical point. The theory proposed by Halperin, Lubenski, and Ma (HLM) [28, 29], taking into account the coupling between $\psi$ and the nematic director fluctuations $\delta \hat{n}$, showed that the $N-\operatorname{Sm} A$ transition is always at least weakly first order which rules out the possibility of a tricritical point. Combining both the $\delta S$ and $\delta \hat{n}$ couplings introduces two more terms in free energy expression as compared to the usual standard form. One term is of the form $\psi^{2} S$ which is nematic-smectic order parameter coupling (referred to as de Gennes coupling) and the other is smectic order-nematic director fluctuation coupling (HLM coupling) $\psi^{2} \delta \hat{n}$. The former coupling reveals the effects of the elasticity of the nematic order prior to the onset of the smectic order and can drive the $N-\operatorname{Sm} A$ transition from XY like to tricritical to weakly first-order[3]. The coupling $\psi^{2} \delta \hat{n}$ causes the anisotropic elastic deformations in the smectic. The strength of this coupling depends on the magnitude of the splay elastic constant $K_{11}$ which is directly proportional to $S^{2}$. Since it is expected that a low-molecular weight solvent miscible in an LC would affect both $\delta S$ and $\delta \hat{n}$ fluctuations, the $x_{\text {sol }}$ dependence would be accounted for using similar terms in a free-energy expansion. 
In this work, the effect of a non-mesogenic, low molecular weight, solvent (n-hexane) concentration on the continuous nematic to smectic- $A(N-\operatorname{Sm} A)$ phase transition on octylcyanobiphenyl (8CB) and n-hexane (hex) binary mixtures (8CB+hex) was studied via highresolution ac-calorimetry as a function of n-hexane concentration, $x_{\text {hex }}$. The introduction of n-hexane on $8 \mathrm{CB}$ causes a dramatic change in the $N$-Sm $A$ phase transition behavior. The heat capacity peaks associated with the $N-\operatorname{Sm} A$ transition, $\delta C_{p}$, shift towards lower temperature non-monotonically and become progressively larger as the hexane concentration increases. The dispersive part of heat capacity $C_{p}^{\prime \prime}$ associated with $N$-Sm $A$ transition has peaks only for higher hexane mole fractions $\left(x_{\text {hex }} \geq 0.08\right)$ but not for the lower hexane mole fractions $\left(x_{\text {hex }} \leq 0.06\right)$ revealing the continuous (second order) nature of the $N-\operatorname{Sm} A$ transition for the lower n-hexane mole fractions $\left(x_{h e x} \leq 0.06\right)$ and first-order nature for higher $n$-hexane mole fractions $\left(x_{h e x} \geq 0.08\right)$. The integrated ac-enthalpy increases overall as a function of hexane molar fraction whereas the imaginary part of the enthalpy reveals a sharp increase at hexane mole fraction of around 0.07 and remains fairly constant. The crossover between continuous to first-order $N-\operatorname{Sm} A$ transition is observed at a tricritical point of $x_{h e x}^{T C P} \approx 0.07$. The non-linear increase in the heat capacity effective critical exponent towards its tricritical value $(\alpha=0.5)$ is observed.

The hysteresis of the $\delta C_{p}$ shape on heating and cooling has been observed is likely due to a microscopic phase separation of the solvent, perhaps into intersticial region between smectic layers. The non-monotonic transition temperature shift may be due to the competing interactions of microphase separation and dilution effects. These effects may also responsible for the $\alpha_{\text {eff }}$ behavior with extended curvature as $x_{\text {hex }} \rightarrow x_{\text {hex }}^{T C P}$. These effects would also have profound consequences on the higher temperature $I-N$ phase transition as well, which was presented in previous paper [30].

This paper is organized as follows; following this introduction, Section II describes the preparation of sample, the calorimetric cell, and the ac-calorimetric procedures employed in this work. Section III describes the calorimetric results and critical behavior of the $N$ $S m A$ phase transition in the $8 \mathrm{CB}+$ hex system. Section IV] discusses these results and draws conclusions. 


\section{EXPERIMENTAL}

The liquid crystal $8 \mathrm{CB}$ has a molecular mass $M_{w}=291.44 \mathrm{~g} \mathrm{~mol}^{-1}$ and a density of $\rho_{L C}=$ $0.996 \mathrm{~g} \mathrm{ml}^{-1}$. The 8CB, purchased from Frinton Lab, was degassed under vacuum for about two hours in the isotropic phase before used for pure and mixture samples. Spectroscopic grade n-hexane (molecular mass of $86.18 \mathrm{~g} \mathrm{~mol}^{-1}$, a density of $0.6548 \mathrm{~g} \mathrm{ml}^{-1}$, and a boiling point of $342 \mathrm{~K}$ ) purchased from EM Science was used without further purification. The 8CB and n-hexane mixtures appear to be miscible up to an n-hexane mole fraction of $\geq 0.12$. This was confirmed by polarizing micrographs of the samples. Measurements were performed on samples as a function of n-hexane mole fraction $x_{\text {hex }}$ ranging from 0 (pure 8CB) to 0.12 .

High resolution ac-calorimetric measurements were carried out using a homemade calorimeter. The calorimetric sample cell consists of an aluminium envelop $15 \times 8 \times 0.5 \mathrm{~mm}$. To prepare an envelop cell, a sheet of aluminum was cleaned using successive application of water, ethanol, and acetone in an ultrasonic bath and then was folded and sealed on three sides with super-glue (cyanoacrylate). Once the cell was thoroughly dried, the desired amount of liquid crystal followed by a relatively large amount of n-hexane were introduced to the cell. The mass of the sample and cell was monitored as the n-hexane was allowed to evaporate slowly until the desired mass of the n-hexane was achieved. At the point of the desired mass of the $8 \mathrm{CB}$ +hex mixture, the envelop flap was quickly folded and sealed with the super-glue. When the filled cell was ready a $120 \Omega$ strain gauge and $1 \mathrm{M} \Omega$ carbon-flake thermistor were attached to opposite surfaces of the cell using GE varnish. The cell was then mounted into the calorimeter, the details of which can be found elsewhere [31 33]. In the ac-mode, oscillating heating power $P_{a c} e^{i \omega t}$ is input to the cell resulting in temperature oscillations with an amplitude $T_{a c}$ and a relative phase shift, $\varphi=\Phi+\pi / 2$, where $\Phi$ is the absolute phase shift between $T_{a c}$ and the input power. Defining the heat capacity amplitude as $C^{*}=P_{a c} /\left(\omega T_{a c}\right)$, the specific heat at a heating frequency $\omega$ can be expressed as

$$
\begin{gathered}
C_{p}=\frac{C_{\text {filled }}^{\prime}-C_{\text {empty }}}{m_{s}}=\frac{C^{*} \cos (\varphi)-C_{\text {empty }}}{m_{s}} \\
C_{p}^{\prime \prime}=\frac{C_{\text {filled }}^{\prime \prime}}{m_{s}}=\frac{C^{*} \sin (\varphi)-\frac{1}{\omega R_{e}}}{m_{s}}
\end{gathered}
$$

where $C_{\text {filled }}^{\prime}$ and $C_{\text {filled }}^{\prime \prime}$ are the real and imaginary parts of the heat capacity, $C_{\text {empty }}$ is the heat capacity of the empty cell, $m_{s}$ is the mass of the sample (in the range of $15 \mathrm{mg}$ to $40 \mathrm{mg}$ ), 
and $R_{e}$ is the external thermal resistance between the cell and the bath. Eq. (11) and (2) need small correction to account the non-negligible internal thermal resistance as compared to $R_{e}$ and this was applied to all samples [34]. The real part of the heat capacity indicates storage (capacitance) of the energy whereas the imaginary part indicates the loss(dispersion) of energy in the sample. Temperatures corresponding to equilibrium, one-phase states exhibit a flat imaginary part of heat capacity, i.e. $C_{p}^{\prime \prime}=0$ [35]. Non-equilibrium dispersive regions, such as a two-phase coexistence region where the latent heat is released, have non-zero $C_{p}^{\prime \prime}$.

Figure 1 illustrates the specific heat capacity variation over an extended temperature range for the $x_{h e x}=0.028 \mathrm{CB}+$ hex sample. The dashed curve under the $N$-Sm $A$ heat capacity peak represents the $I$ - $N$ specific heat capacity wing $C_{p}^{\text {wing }}$ expected in the absence of the $N-\operatorname{Sm} A$ transition. This wing is used to determine the excess specific heat associated with the $N-\operatorname{Sm} A$ phase transition

$$
\delta C_{p}=C_{p}-C_{p}^{w i n g}
$$

The enthalpy change associated with a phase transition is defined as

$$
\delta H=\int \delta C_{p} d T
$$

For a second-order or continuous phase transition, the limits of integration are as wide as possible about the $\delta C_{p}$ peak and gives the total enthalpy change $(\delta H)$ associated with the transition. But for a first-order transition the situation is complicated due to the presence of a coexistence region as well as a latent heat $\Delta H$. The total enthalpy change for a weakly first order phase transitions is the sum of the integrated enthalpy and the latent heat, $\Delta H_{\text {total }}=\delta H+\Delta H$. Due to partial phase conversion during a $T_{a c}$ cycle, typical $\delta C_{p}$ values obtained in the two-phase coexistence region are artificially high and frequency dependent. A simple integration of the observed $\delta C_{p}$ peak yields an effective enthalpy change $\delta H^{*}$ for the first-order transition which includes some of the latent heat contribution. If we integrate the imaginary part of heat capacity given by Eq. (2), we can get the imaginary transition enthalpy $\delta H^{\prime \prime}$, which is the dispersion of energy in the sample and is a proxy of latent heat associated with the transition. In an ac-calorimetric technique the uncertainty in determining the enthalpy is typically $10 \%$ due to the uncertainty in the baseline and background subtraction. 


\section{RESULTS}

\section{A. The $N-\operatorname{Sm} A$ Heat Capacity}

The resulting $\delta C_{p}$ data of the $N$-Sm $A$ transition on heating for $8 \mathrm{CB}$ +hex and pure $8 \mathrm{CB}$ samples over a $\pm 1.5 K$ temperature range window about the $\delta C_{p}$ peak is shown in Fig. 2 (upper panel). As the mole fraction of n-hexane increases, the $N$-Sm $A$ heat capacity peak becomes larger than the pure $N$-Sm $A$ peak and with apparently larger wings on the high temperature side of the peak. Figure 2 (lower panel) shows the imaginary part of specific heat $C_{p}^{\prime \prime}$ on heating as a function of temperature about $T_{N A}$. For the n-hexane mole fractions $x_{h e x} \leq 0.06$, the $C_{p}^{\prime \prime}$ is flat, indicating the second-order nature of the transition. For $x_{\text {hex }} \geq 0.08$, the $C_{p}^{\prime \prime}$ reveals a peak indicating a first-order behavior of the transition. As the mole fraction of n-hexane increases beyond $x_{\text {hex }} \geq 0.08$, the $C_{p}^{\prime \prime}$ peak become broader with a two-phase co-existence region growing from $\sim 0.35 \mathrm{~K}$ at $x_{\text {hex }}=0.08$ to $\sim 0.85 \mathrm{~K}$ at $x_{\text {hex }}=0.12$.

The $N$-Sm $A$ excess specific heat $\delta C_{p}$ (upper panel) and imaginary part of heat capacity $C_{p}^{\prime \prime}$ (lower panel)on cooling are shown in Fig. 3. On cooling, the $\delta C_{p}$ peaks exhibit larger $C_{p}$ wings on both sides of $T_{N A}$ but the low temperature wing appears progressively smeared in temperature. In addition, the $\delta C_{p}$ on cooling exhibits sharp peaks up to $x_{h e x}=0.08$ then appears rounded for $x>0.08$. The $N$-Sm $A C_{p}^{\prime \prime}$ behavior on cooling is similar to the heating scans in that $C_{p}^{\prime \prime}=0$ through $T_{N A}$ for $x_{\text {hex }} \leq 0.06$, then reveals a peak for $x_{\text {hex }} \geq 0.08$. This indicates, as on heating, a cross-over from continuous to first-order transition behavior. However, the $C_{p}^{\prime \prime}$ peaks for $x_{h e x} \geq 0.08$ on cooling have markedly different shape than on heating. Here, as the temperature approaches $T_{N A}$ from above, a sharp jump preceded by a relatively small wings occurs at $\sim 0.1 \mathrm{~K}$ above $T_{N A}$ for all $8 \mathrm{CB}+$ hex samples. As the temperature cools further, a long $C_{p}^{\prime \prime}$ tail is seen to a common trend at $\sim-0.25 K$ for $x_{\text {hex }}=0.08$ and $\sim-0.4 K$ for $x_{\text {hex }}=0.09$ and 0.12 below $T_{N A}$. The increase in the two-phase co-existence is similar to that seen on heating.

The $N$-Sm $A$ transition temperature $T_{N A}$ is defined as the temperature of the $\delta C_{p}$ peak maximum and the $I-N$ transition temperature is taking at the lowest temperature of the isotropic phase prior to entering the $I+N$ two-phase coexistence region[30]. Figure 4 (upper panel) shows the $I-N$ and $N$-Sm $A$ phase transition temperatures as a function of $x_{\text {hex }}$. As 
$x_{\text {hex }}$ increases, both transition temperatures decrease non-linearly with a bump at $x_{\text {hex }} \sim$ 0.07. Figure 4 (lower panel) shows the nematic temperature range $\Delta T_{N}=T_{I N}-T_{N A}$ as a function of $x_{\text {hex }}$ revealing a similar non-linear trend with a similar bump at the same $x_{h e x}$. The horizontal dashed, dashed dot, and dot lines represent nematic ranges for pure 9CB[36], $8 \mathrm{CB}+$ chex [19], and 8CB $+10 \mathrm{CB}$ [15] at the tricritical point respectively. The solid straight lines are the transition temperatures (Fig. 4-upper panel) and nematic range (Fig. 4-lower panel) for the 8CB+chex system [19]

Since continuous transition behavior is observed for $x_{\text {hex }}=0.06$ and first-order behavior at $x_{\text {hex }}=0.08$, a tricritical point mole fraction is taken as $x_{h e x}^{T C P}=0.07$ with the corresponding nematic range at $\Delta T_{N}^{T C P} \simeq 4.63 \mathrm{~K}$. The vertical dashed line in both the panels of Fig. 4 indicates $x_{h e x}^{T C P}$ and a bold-bordered box in the lower-panel gives the location of the crossover point whose width and height are the magnitude of uncertainties in $x_{h e x}^{T C P}$ and $\Delta T_{N}^{T C P}$ respectively.

The effective $N$-Sm $A$ transition enthalpy $\delta H_{N A}^{*}$ was obtained by integrating $\delta C_{p}$ in the range $\pm 3 \mathrm{~K}$ about $T_{N A}$. The dispersive enthalpy, $\delta H_{N A}^{\prime \prime}$ of the $N$-Sm $A$ transition, available only for $x_{\text {hex }} \geq 0.08$, and was obtained by integrating the $N-\operatorname{Sm} A C_{p}^{\prime \prime}$ peak. Since a fixed heating frequency was used, the non-zero $\delta H_{N A}^{\prime \prime}$ is only proportional to the transition latent heat. The resulting $\delta H_{N A}^{*}$ and $\delta H_{N A}^{\prime \prime}$ for heating (०) and cooling (•) scans as a function of $x_{h e x}$ for all $8 \mathrm{CB}+$ hex samples are shown in Fig. 5. The $\delta H_{N A}^{*}$ values show an overall increase in value with increasing $x_{h e x}$ and are consistent on heating and cooling. A small apparent jump in $\delta H_{N A}^{*}$ is seen at $\sim x_{h e x}^{T C P}$. See Figure 5(upper panel). The $\delta H_{N A}^{\prime \prime}$ exhibits a sudden jump from 0 to $\sim 0.28 \mathrm{~J} / \mathrm{g}$ at $x_{h e x}^{T C P}$.

A summary of these results for $8 \mathrm{CB}+$ hex samples including pure $8 \mathrm{CB}$ is tabulated in Table I. Included are the n-hexane molar fraction $x_{h e x}$, the $N$-Sm $A$ transition temperatures $T_{N A}$, nematic range $\Delta T_{N}$, integrated enthalpy change $\delta H_{N A}^{*}$, imaginary enthalpy $\delta H_{N A}^{\prime \prime}$, McMillan ratio $M R$ and height of excess heat capacity peaks $h_{M}$ for all the $8 \mathrm{CB}+$ hex samples including pure 8CB.

\section{B. Power-law Analysis of $N-\operatorname{Sm} A$ phase transition}

Because the $\delta C_{p}$ for the $N$-Sm $A$ transition in $8 \mathrm{CB}+$ hex remains continuous and sharp for $x_{\text {hex }} \leq x_{h e x}^{T C P}$, a critical power-law analysis was performed. The usual power law form in 
terms of reduced temperature, $|t|=\left|\left(T-T_{c}\right)\right| / T_{c}$, that is used to analyze the excess specific heat associated with $N$-Sm $A$ transition is given by [18]

$$
\delta C_{p}=A^{ \pm}|t|^{-\alpha}\left(1+D_{1}^{ \pm}|t|^{\Delta_{1}}\right)+B_{c}
$$

where $B_{c}$ is the critical background, $A^{ \pm}$are the amplitudes above and below the transition, $D_{1}^{ \pm}$are the correction-to-scaling amplitude with an exponent $\Delta_{1}=0.524$ [18]. A full, non-linear, fitting of Eq.(5) to the $\delta C_{p}$ data was attempted, but because the number of data close to the peak were relatively sparse, these fits did not properly converge.

A simple power-law analysis procedure was employed in order to estimate the variation of the critical exponent $\alpha$ as a function of $x_{h e x}$. This procedure begins by approximating $T_{c}$ for each continuous $\delta C_{p}$ peak. This is done by plotting a $\log \left(\delta C_{p}\right)$ vs $\log (|t|)$ and choosing $T_{c}$ such that the high and low temperature wings appear linear and parallel to each other for low $|t|$. The rounded and non-power-law data points are easily determined and removed.

Figure 6] shows the resulting log-log plot of data above and below $T_{c}$ for pure $8 \mathrm{CB}\left(x_{\text {hex }}=\right.$ 0 ) and the highest concentration $8 \mathrm{CB}+$ hex sample that is continuous as determined by $C_{p}^{\prime \prime}$ $\left(x_{h e x}=0.06\right)$. Now, a range of data up to $\left|t_{\max }\right|$ was chosen in order to perform a simple linear fit, $\log \left(\delta C_{p}\right)=\log \left(A^{ \pm}\right)-\alpha_{\text {eff }}^{\prime} \log |t|$. Here, $\left|t_{\text {max }}\right|$ varied smoothly from $8.9 \times 10^{-4}$ for pure $8 \mathrm{CB}$ to $1.8 \times 10^{-3}$ for the $x_{\text {hex }}=0.068 \mathrm{CB}+$ hex sample. The resulting linear fits are shown in Fig. 6 for data above and below $T_{c}$. The difference between $\alpha_{\text {eff }}^{\prime}\left(T>T_{c}\right)$ and $\alpha_{e f f}^{\prime}\left(T<T_{c}\right)$ is taken as the uncertainty in $\alpha_{e f f}^{\prime}$. The resulting $\alpha_{e f f}^{\prime}$ are not the true critical exponents because of this simplified analysis. However, comparing the pure $8 \mathrm{CB}$ result here to the literature value of $\alpha_{\text {eff }}=0.3$ [3] , a corrected $\alpha_{\text {eff }}$ for the $8 \mathrm{CB}+$ hex samples is taken as an algebraic shift of +0.17 , which is the difference of $\alpha_{\text {eff }}^{\prime}-\alpha_{\text {eff }}$ for pure $8 \mathrm{CB}$. This procedure was applied for all samples from $x_{\text {hex }}=0$ to 0.06 and should reasonably approximate the $x_{\text {hex }}$ dependence of $\alpha_{\text {eff }}$.

The resulting estimate of the $N-\operatorname{Sm} A$ heat capacity effective critical exponent as a function of $x_{h e x}$ are shown in Fig. 7. Here, a linear rapid rise in $\alpha_{e f f}$ is seen as $x_{h e x}$ increases from 0 to 0.04 then curving over for $x_{\text {hex }}>0.04$. The upward arrow in Fig. 7 is the best estimate of $x_{h e x}^{T C P}$ for this $8 \mathrm{CB}+$ hex system. 


\section{DISCUSSION AND CONCLUSION}

The continuous $N$-Sm $A$ liquid crystal phase transition has been studied using highresolution ac-calorimetry as a function of solvent dopant concentration. Multiple heating and cooling cycles reproduce each other for $x_{h e x} \leq 0.12$ along with no visual indication of phase separation support the view that the $8 \mathrm{CB}+$ hex binary system remained mixed (nhexane miscible) for all samples studied here, without mechanical mixing. This is supported also by x-ray studies of the smectic layer spacing in $8 \mathrm{CB}$ +hex that showed phase separation for $x_{\text {hex }}>1.0$ [23]. In this work, the smectic layer spacing increased with increasing $x_{h e x}$ and was interpreted as a nano-scale partitioning of n-hexane in between smectic layers.

A more recent calorimetric study of binary mixtures of $8 \mathrm{CB}$ with various, low-molecular weight, solvents found dramatic changes to the character of the $N$-Sm $A$ phase transition [19]. In this work, the $N$-Sm $A$ transition approaches a tricritical point linearly. However, this study used cyclo-hexane, that has ring structure and employed continuous mixing during measurements as a function of cyclohexane mole-fraction, $x_{\text {chex }}$. The transition temperature $T_{N A}$ decreases linearly as $x_{\text {chex }}$ increases, the critical exponent $\alpha$ increases linearly from 0.31 (pure 8CB) to 0.50 at $x_{\text {chex }}=0.046$, and the onset of a $N$-Sm $A$ latent heat occurs smoothly

at TCP, $x_{\text {chex }}^{T C P}=0.046$. These results were modelled using mean-field Landau-deGennes theory incorporating the nematic free-energy, smectic free-energy, and a coupling between nematic and smectic order parameters. This model was extended to account for the solvent by adding a solvent mole-fraction coupling to $\psi^{2}$ and to $\psi^{2} \delta S$ to the total solvent free-energy.

Similar results were found in 8CB+biphenyl binary mixtures and a Landau-de Gennes model that accounted for change in the LC elastic constants with $x_{\text {sol }}$. However, TCP was not found in $8 \mathrm{CB}+$ biphenyl system [24].

In this present study, several important differences emerge. As $x_{h e x}$ increases, $T_{N A}$ decreases as well as the nematic range $\Delta T_{N}$ in a non-linear way. The character of $N-\operatorname{Sm} A$ transition remains continuous up to $x_{\text {hex }} \simeq 0.07$ where it appears to jump suddenly to a first-order transition. The bump in $T_{N A}$ and $\Delta T_{N}$ as well as the jump in $\delta H_{N A}^{\prime \prime}$ all occur at $x_{\text {hex }}^{T C P}$.

The critical behavior, estimated by the simple power-law analysis presented here, evolves with $\alpha_{\text {eff }}$ initially increases linearly as in the $8 \mathrm{CB}+$ chex system but then curves over to reach $\alpha_{\text {eff }}=0.50$ at $x_{\text {hex }} \rightarrow x_{h e x}^{T C P}$. Qualitatively, the correction-to-scaling terms $D^{ \pm}$and 
the amplitude ratio $A^{-} / A^{+}$are changing their values towards the tricritical values as a function of $x_{h e x}$. Here, the qualitative measurement of the amplitude ratio $A^{-} / A^{+}$was

extracted examining the gap between two slope lines of the linear fit of log-log plot of $\delta C_{p}$ vs $|t|$ (Fig. 6) and the curvature of the curve at high $|t|$ was observed to get qualitative measure of $D^{ \pm}$.

The addition of n-hexane in $8 \mathrm{CB}$ creates the random dilution effect which causes the decrease in transition temperature $T_{N A}$ and nematic range $\Delta T_{N A}$. The experiment was done without stirring the sample which may cause some phase separation in microscopic or even in nanoscopic scale. These dilution and microphase separation effects, may cause to develop the two competing interactions which cause the non-linearity in the transition temperature $T_{N A}$, nematic range $\Delta T_{N}$, effective critical exponent $\alpha_{\text {eff }}$ and jump in the imaginary enthalpy $\delta H_{N A}^{\prime \prime}$. These effects also cause the change in coupling between the order parameters $\psi$ and $Q$ which consequently change the order of the $N$-Sm $A$ phase transition from continuous to first order with a critical point at $x_{h e x}^{T C P} \simeq 0.07$.

We have undertaken a detailed calorimetric studies on the effect of non- mesogenic, low molecular weight solvent(hexane) on octylcyanobiphenyl(8CB) phase transitions with emphasis on the most extensively studied but controversial $N$-Sm $A$ phase transition. The addition of the hexane on $8 \mathrm{CB}$ dilutes the mixture and changes the intermolecular potential. The microscopic phase separation and dilution effect due to the introduction of n-hexane in $8 \mathrm{CB}$ cause the change in magnitude of $S-\psi$ and $\psi-\delta \hat{n}$ couplings which consequently change the phase transition behavior. The result obtained reveals new aspect of the effect of non-mesogenic disorder on the liquid crystal transition.

\section{Acknowledgments}

This work was supported by the Department of Physics at WPI. 
[1] P. G. de Gennes and J. Prost, The Physics of Liquid Crystals (Oxford University Press, Clarendon, Oxford, England, 1993).

[2] S. Chandrashekhar, Liquid Crystals (Cambridge University Press, England, 1992).

[3] G. S. Iannacchione, S. Park, C. W. Garland, R. J. Birgeneau, and R. L. Leheny, Phys. Rev. E 67, 011709 (2003).

[4] G. S. Iannacchione, Fluid Phase Equilibria 222/223, 177 (2004).

[5] F. Cruceanu, D. Liang, R. L. Leheny, C. W. Garland, and G. S. Iannacchione, Phys. Rev. E 79, 011710 (2009).

[6] L. Wu, B. Zhou, C. W. Garland, T. Bellini, and D. W. Schaefer, Phys. Rev. E 51, 2157 (1995).

[7] T. Bellini, L. Radzihosky, J. Toner, and N. A. Clark, Science 294, 1074 (2001).

[8] A. Zidansek, G. Lahajnar, and S. Kralj, Appl. Magn. Reson. 27, 311 (2004).

[9] Z. Kutnjak, S. Kralj, G. Lahajnar, and S. Zumer, Phy. Rev. E 68, 021705 (2003).

[10] Z. Kutnjak, S. Kralj, G. Lahajnar, and S. Zumer, Fluid Phase Equilibria 222-223, 275 (2004).

[11] R. Mukhopadhyay, A. Yethiraj, and J. Bechhoefer, Phys. Rev. Lett. 83, 4796 (1999).

[12] A. Primak, M. Fisch, and S. Kumar, Phys. Rev. Lett. 88, 035701 (2002).

[13] K. J. Stine and C. W. Garland, Phys. Rev. A 39, 3148 (1989).

[14] M. B. Sied, J. Salud, D. O. Lopez, M. Barrio, and J. L. Tamarit, Phys. Chem. Chem. Phys. 4, 2587 (2002).

[15] M. G. Lafouresse, M. B. Sied, H. Allouchi, D. O. Lopez, J. Salud, and J. L. Tamarit, Chem. Phys. Lett. 376, 188 (2003).

[16] M. B. Sied, D. O. Lopez, J. L. Tamarit, and M. Barrio, Liquid Crystals 29, 57 (2002).

[17] S. Jeong, D. Kim, K. Rhie, M. Hong, S. Kumar, W. Jang, , and S. Shin, Journal of Physics D: Applied Physics 41, 062002 (2008).

[18] C. W. Garland and G. Nounesis, Phys. Rev. E 49, 2964 (1994).

[19] K. Denolf, G. Cordoyiannis, C. Glorienx, and J. Thoen, Phys. Rev. E 76, 051702 (2007).

[20] K. Denolf, B. V. Roie, C. Glorienx, and J. Thoen, Phys. Rev. Lett. 97, 107801 (2006).

[21] P. K. Mukherjee, J. Chem. Phys. 116, 9531 (2002).

[22] S. DasGupta and S. K. Roy, Phys. Lett. A 288, 323 (2001).

[23] T. P. Rieker, Liquid Crystals 19, 497 (1995). 
[24] S. DasGupta and P. C. ans Soumen Kumar Roy, Phys. Rev. E 63, 041703 (2001).

[25] A. Yethiraj, R. Mukhopadhyay, and J. Bechhoefer, Phys. Rev. E 65, 021702 (2002).

[26] C. W. Garland and G. S. Iannacchione, J. Phys. Chem. B 113, 3901 (2008).

[27] W. L. McMillan, Phys. Rev. A 4, 1238 (1971).

[28] B. Halperin, T. C. Lubensky, and S. K. Ma, Phys. Rev. Lett. 32, 292 (1974).

[29] M. Anisimov, P. Cladis, E. E. Gorodetskii, D. A. Huse, V. E. Podneks, V. Taratuta, W. van Saarloos, and V. P. Voronov, Phys. Rev. A 41, 6749 (1990).

[30] K. P. Sigdel and G. S. Iannacchione, Submitted to J. chem. Phys. (2010).

[31] P. F. Sullivan and G. Seidel, Phys. Rev. 173, 679 (1968).

[32] D. Finotello, S. Qian, and G. S. Iannacchione, Thermochimica Acta 304/305, 303 (1997).

[33] H. Yao and C. W. Garland, Rev. Sci. Instrum. 69, 172 (1998).

[34] A. Roshi, G. S. Iannacchione, P. S. Clegg, and R. J. Birgeneau, Phys. Rev. E 69, 031703 (2004).

[35] G. S. Iannacchione, C. W. Garland, J. T. Mang, and T. P. Rieker, Phys. Rev. E 58, 5966 (1998).

[36] S. Qian, G. S. Iannacchione, and D. Finotello, Phys. Rev. E 57, 4305 (1998). 
Figures 


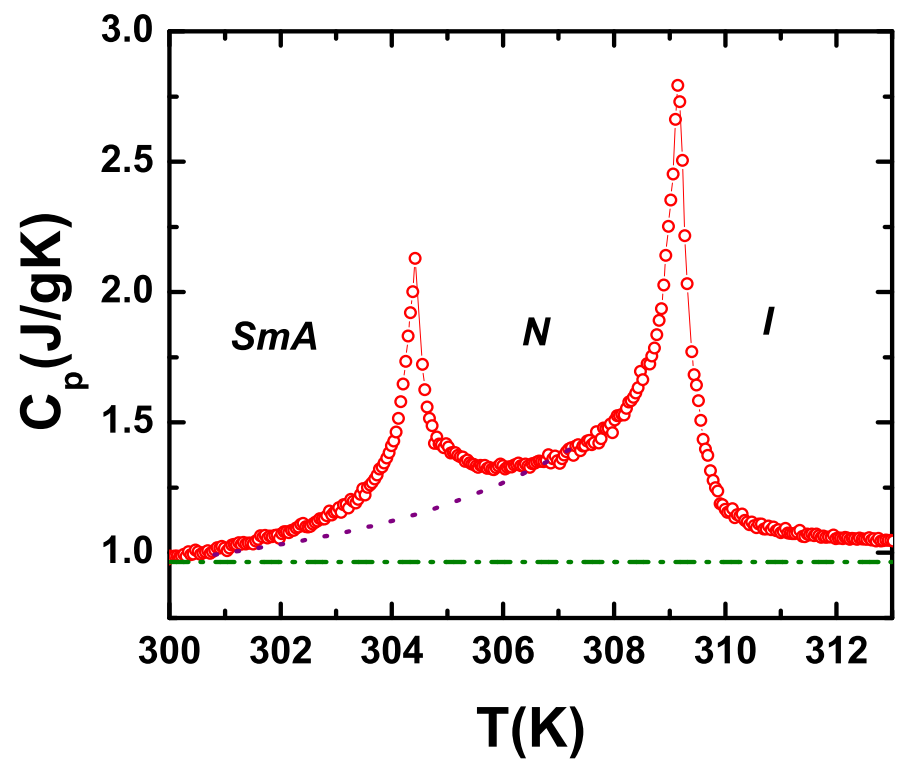

FIG. 1: Specific heat capacity for an $8 \mathrm{CB}+$ hex sample on heating with $x_{h e x}=0.02$. The dashed dotted line represents the $C_{p}$ background, while the dashed curve acts as $C_{p}^{\text {wing }}$ and represents the low temperature $I-N C_{p}$ wing that would be expected in the absence of $N$-Sm $A$ transition. 


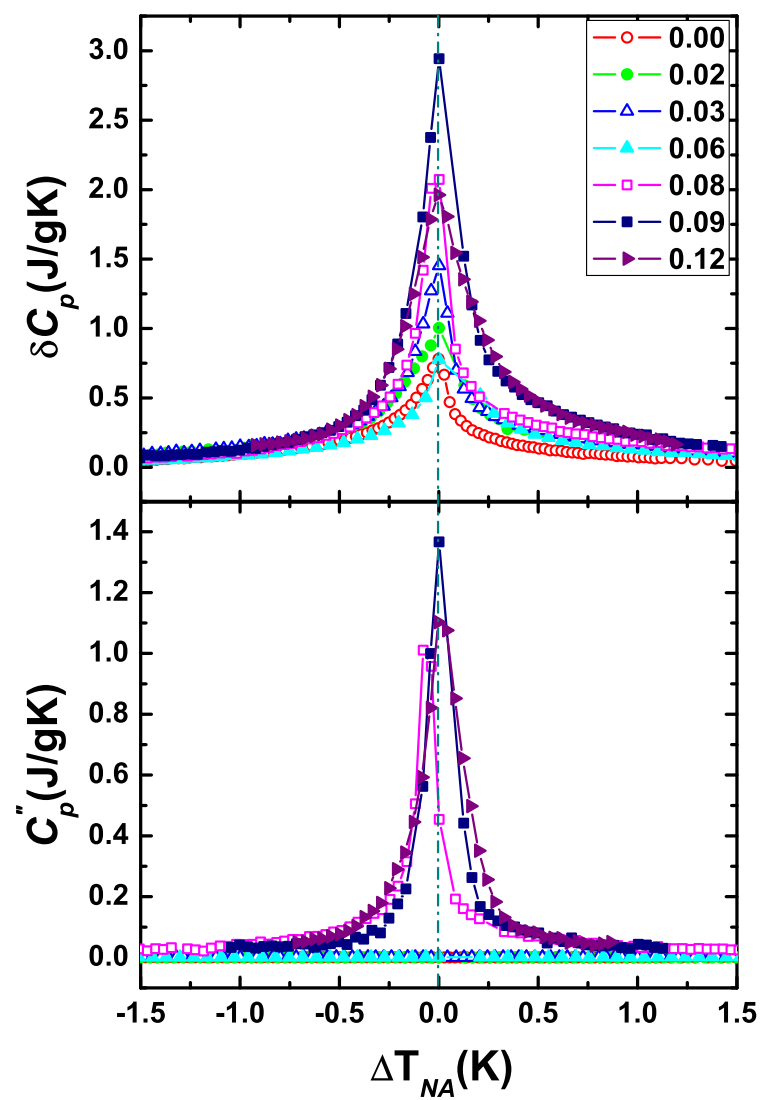

FIG. 2: Upper panel: The excess specific heat $\delta C_{p}$ associated with the $N$-Sm $A$ transition on heating as a function of temperature about $T_{N A}$ for pure and all $8 \mathrm{CB}+$ hex samples. See legend. Lower panel: The imaginary part of heat capacity on heating for all samples as a function of temperature about $T_{N A}$. 


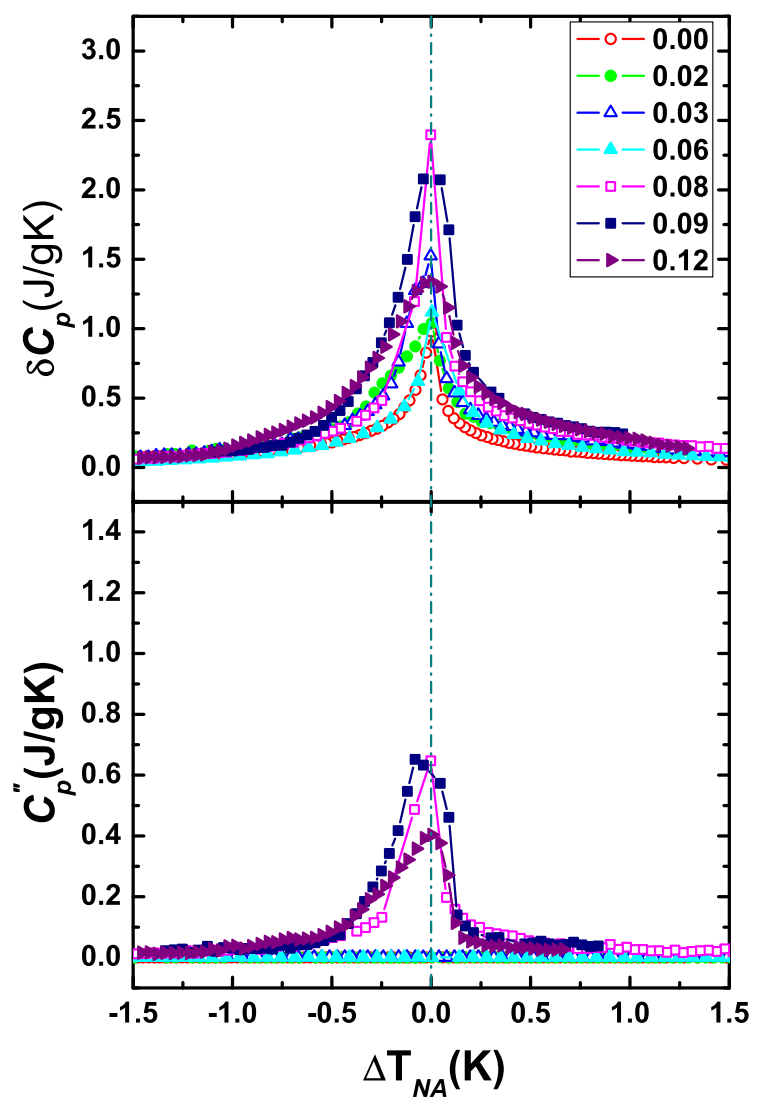

FIG. 3: Upper panel: The excess specific heat $\delta C_{p}$ associated with the $N$-Sm $A$ transition on cooling as a function of temperature about $T_{N A}$ for pure and all $8 \mathrm{CB}+$ hex samples. The definition of the symbols are given on the inset. Lower panel: The imaginary part of heat capacity on cooling for all samples as a function of temperature about $T_{N A}$. 


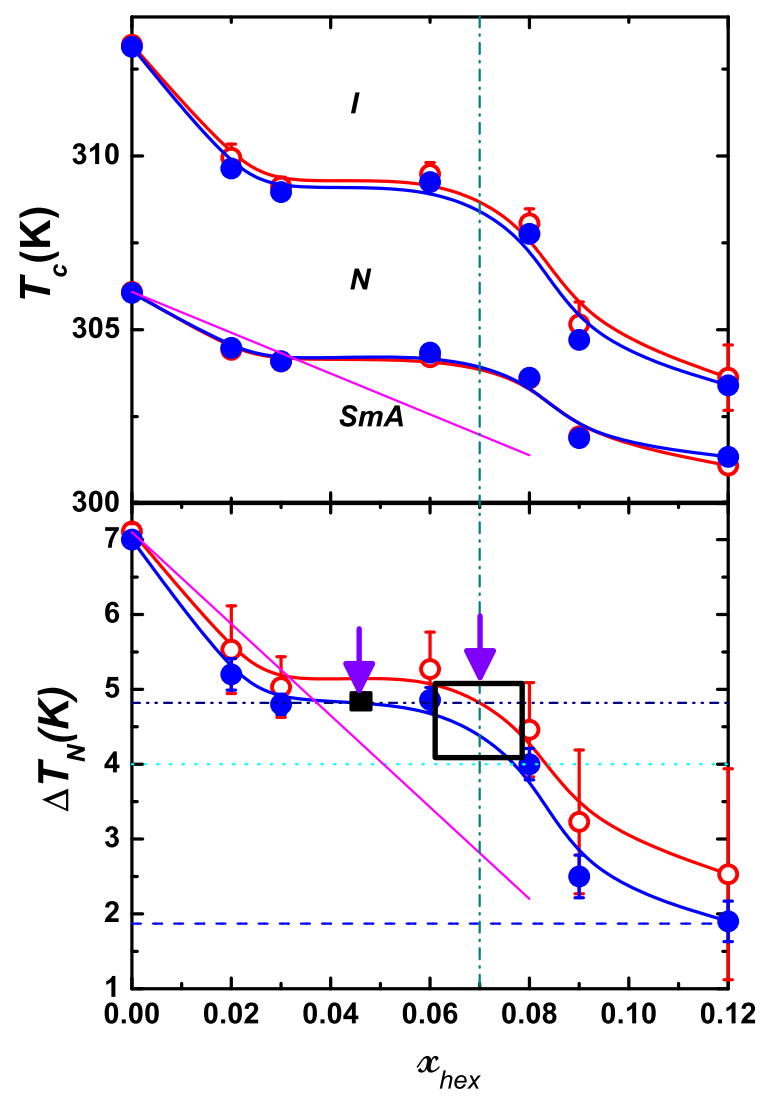

FIG. 4: Upper Panel: The $I-N$ and $N-\operatorname{Sm} A$ phase transition temperatures on heating (o) and cooling $(\bullet)$ as a function of $x_{h e x}$. Solid lines are guides to the eye. Lower panel: The nematic temperature range $\Delta T_{N}$ on heating (o) and cooling $(\bullet)$ as a function of $x_{h e x}$. The closed and open rectangular boxes represent the position of tricritical points for $8 \mathrm{CB}+$ chex [19] and $8 \mathrm{CB}+$ hex systems respectively and the width and height of the boxes represent the uncertainties on $x_{h e x}^{T C P}$ and $\Delta T_{N}^{T C P}$ respectively. The horizontal dashed, dashed dot, and dot lines are nematic ranges for pure $9 \mathrm{CB}[36], 8 \mathrm{CB}+$ chex, and $8 \mathrm{CB}+10 \mathrm{CB}$ [15] respectively at tricritical point. The solid straight lines are transition temperature (upper panel) and nematic range (lower panel) for $8 \mathrm{CB}+$ chex system [19]. 


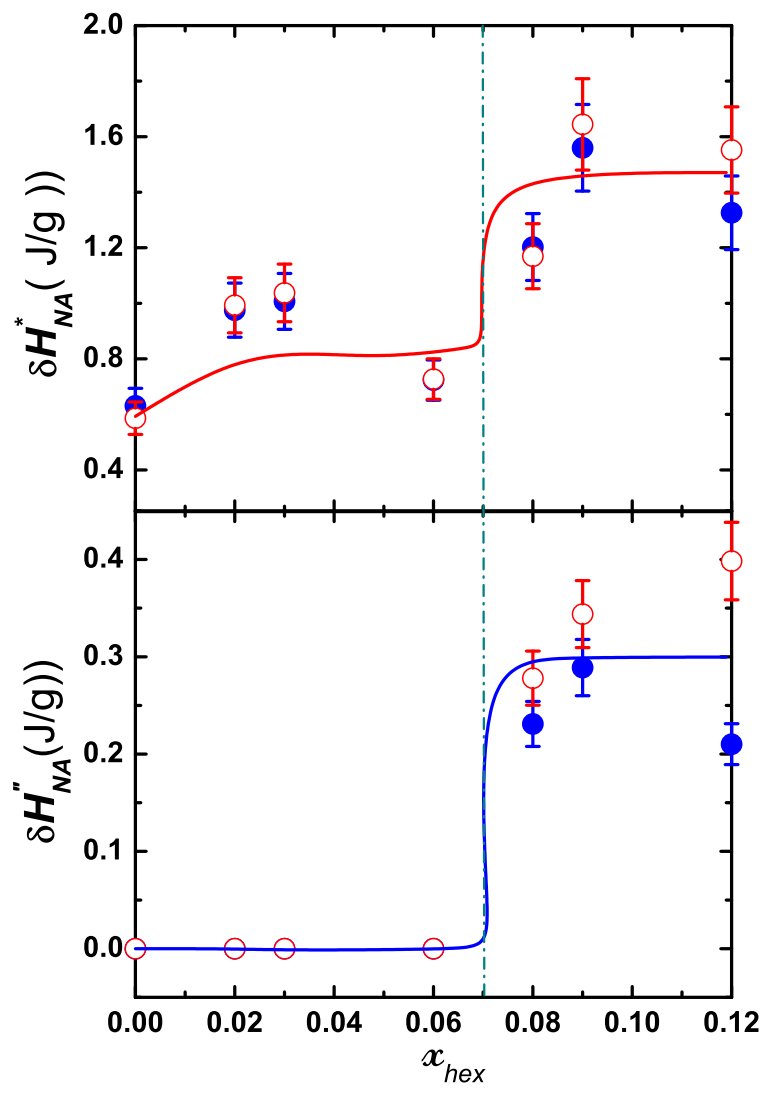

FIG. 5: Upper panel: The total integrated $\delta C_{p}$ ac-enthalpy $\delta H_{N A}^{*}$ on heating (०) and cooling (•) as the function of $x_{h e x}$. Lower panel: Integrated $C_{p}^{\prime \prime}$ enthalpy $\delta H_{N A}^{\prime \prime}$ on heating (o) and cooling (•) as the function of $x_{h e x}$. Solid lines are guides to the eye. 


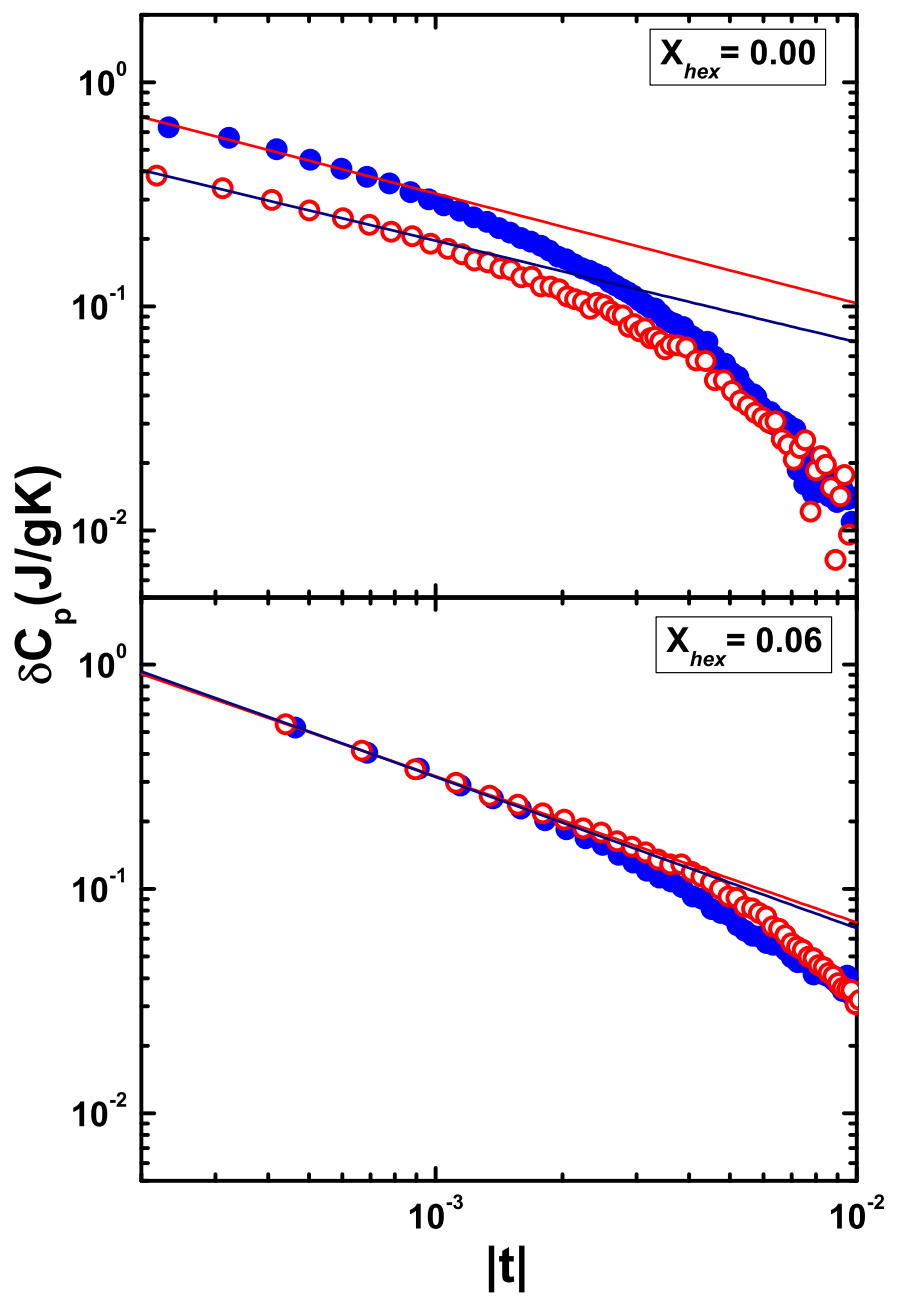

FIG. 6: Upper panel: Excess specific heats associated to $N$-Sm $A$ phase transition as a function of reduced temperature for pure $8 \mathrm{CB}$ for $T<T_{c}(\bullet)$ and for $T>T_{c}(\circ)$. Lower panel: Excess specific heats associated to $N$-Sm $A$ phase transition as a function of reduced temperature for hexane mole fraction $x_{h e x}=0.06$ for $T<T_{c}(\bullet)$ and for $T>T_{c}(\circ)$. Slope of the straight line in each graph gives the effective critical exponent $\alpha_{e f f}$. 


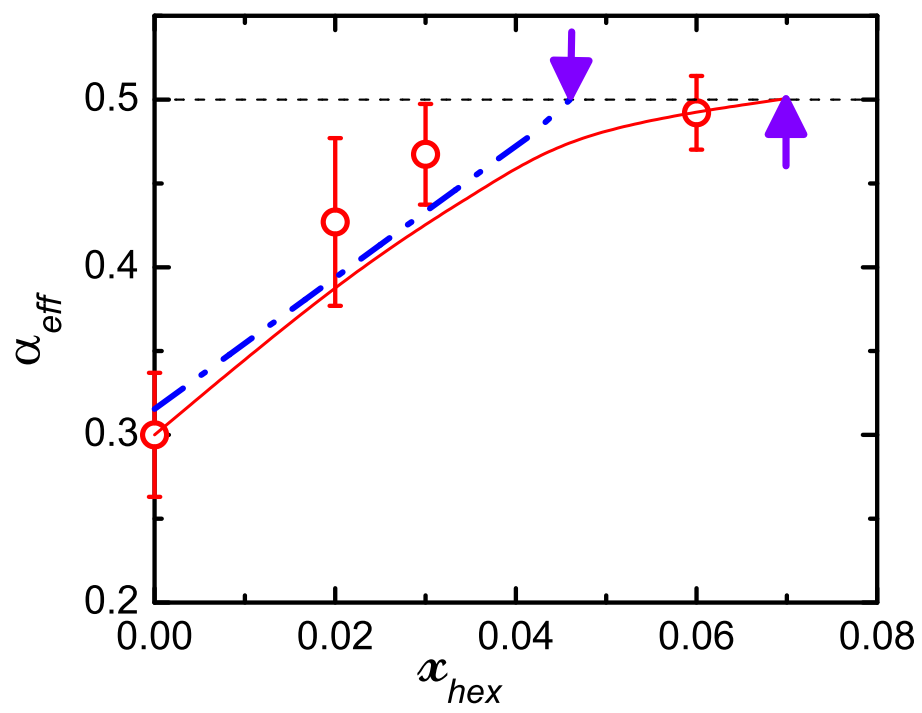

FIG. 7: The effective critical exponent as a function of hexane mole fraction. solid line represents the best estimate of $\alpha_{e f f}$ as a function of $x_{h e x}$. This line intersects the horizontal dashed line at the tricritical point, $x_{\text {hex }} \simeq 0.07$. The dashed-dot line is $\alpha_{\text {eff }}$ for $8 \mathrm{CB}+$ chex system from reference 19]. Vertical arrows indicate the location of the tricritical points for $8 \mathrm{CB}+$ chex (downward arrow) [19] and $8 \mathrm{CB}+$ hex(upward arrow). 
Tables 
TABLE I: Summary of the calorimetric results for pure and all $8 \mathrm{CB}+$ hex samples on heating. Shown are hexane molar fraction $x_{h e x}, N-\operatorname{Sm} A$ transition temperature $T_{N A}$, nematic range $\Delta T_{N}=$ $T_{I N}-T_{N A}$ ( in Kelvin), integrated enthalpy change $\delta H_{N A}^{*}$, imaginary enthalpy $\delta H_{N A}^{\prime \prime}$ (in $\mathrm{J} / \mathrm{g}$ ), McMillan's Ratio $M R$, and heat capacity maximum $h_{M} \approx \delta C_{p}^{\max }(N-A)$ in $\left(J K^{-1} g^{-1}\right)$.

\begin{tabular}{ccccccc}
\hline \hline$x_{\text {hex }}$ & $T_{N A}$ & $\Delta T_{N}$ & $\delta H_{N A}^{*}$ & $\delta H_{N A}^{\prime \prime}$ & $M R$ & $h_{M}$ \\
\hline 0.00 & $306.09 \pm 0.06$ & $7.11 \pm 0.11$ & $0.59 \pm 0.06$ & - & 0.977 & 0.78 \\
0.02 & $304.42 \pm 0.09$ & $5.53 \pm 0.59$ & $0.99 \pm 0.10$ & - & 0.982 & 1.00 \\
0.03 & $304.09 \pm 0.08$ & $5.03 \pm 0.41$ & $1.04 \pm 0.10$ & - & 0.984 & 1.45 \\
0.06 & $304.21 \pm 0.14$ & $5.27 \pm 0.50$ & $0.73 \pm 0.07$ & - & 0.983 & 0.78 \\
0.08 & $303.60 \pm 0.16$ & $4.46 \pm 0.63$ & $1.17 \pm 0.12$ & $0.28 \pm 0.03$ & 0.986 & 2.07 \\
0.09 & $301.93 \pm 0.17$ & $3.23 \pm 0.96$ & $1.64 \pm 0.16$ & $0.29 \pm 0.03$ & 0.989 & 2.94 \\
0.12 & $301.09 \pm 0.08$ & $2.53 \pm 1.41$ & $1.55 \pm 0.15$ & $0.40 \pm 0.04$ & 0.989 & 1.96 \\
\hline \hline
\end{tabular}

